\title{
Design of the Mainz Active Polarized Proton Target
}

\author{
M. Biroth ${ }^{* a}$, P. Achenbach ${ }^{a}$, E. Downie ${ }^{b}$, A. Thomas ${ }^{a}$ \\ for the A2 Collaboration \\ ${ }^{a}$ Institut für Kernphysik, Johannes Gutenberg-Universität, Mainz, Germany \\ ${ }^{b}$ Physics Department, George Washington University, Washington DC, USA \\ E-mail: biroth@kph.uni-mainz.de, \\ patrickakph.uni-mainz.de, \\ edownielgwu.edu, \\ thomas@kph.uni-mainz.de
}

\begin{abstract}
At the MAMI electron accelerator in Mainz, Germany, the A2 Collaboration investigates the spin-polarizabilities of the proton by scattering experiments with spin-polarized energy-tagged photons. An active polarized proton target was developed to identify the reactions by detecting recoil protons inside the Mainz-Dubna dilution cryostat. Polarizable plastic scintillator disks are stacked in a target head made of wavelength-shifting material or of PMMA. Material and geometry of the head determine its scintillation light output. The light is then guided through a tube of borosilicate glass to silicon photomultipliers. All parts of the active polarized proton target are operated at cryogenic temperatures.
\end{abstract}

XVIth International Workshop in Polarized Sources, Targets, and Polarimetry, PSTP2015,

14-18 September 2015,

Bochum, Germany

\footnotetext{
* Speaker.
} 


\section{Introduction}

At the MAMI electron accelerator in Mainz, Germany, the A2 Collaboration investigates the spin-polarizabilities of the proton by scattering experiments with spin-polarized energy-tagged photons. Due to the excellent temperature stability of the Mainz Frozen Spin Target a large degree of proton polarization with high relaxation times can be achieved.

At the core of the frozen spin target for the Crystal Ball detector at MAMI is a roughly $2 \mathrm{~m}$ long, horizontal ${ }^{3} \mathrm{He} /{ }^{4} \mathrm{He}$ dilution refrigerator that was built in cooperation with the Joint Institute for Nuclear Research (JINR) Dubna. The cryostat has a separator working at $3 \mathrm{~K}$ and an evaporator working at $1.2 \mathrm{~K}$ in the pre-cooling stages. At the target position the cryostat provides a very low operation temperature of $25 \mathrm{mK}$.

The forming of highly polarized target nuclei is a two step process: in the initial step a high degree of nucleon polarization is achieved through a microwave pumping process, known as Dynamic Nucleon Polarization (DNP). This requires placing the target material in a highly uniform magnetic field of typically 2.5 Tesla and passing microwave radiation at a frequency near $70 \mathrm{GHz}$ through it. The use of the microwaves leads to a moderate increase of the base temperature of the cryostat from $0.02 \mathrm{~K}$ to around $0.2 \mathrm{~K}$. In a second step, the microwaves are switched off. Consequently, the temperature of the target material drops and the relaxation time of the nucleons increases to somewhere in the order of several thousand hours, although the field is reduced to a holding field of only $0.68 \mathrm{~T}$ for the longitudinal polarization and $0.5 \mathrm{~T}$ for the transverse polarization. Then a measurement period of up to approximately one week in the frozen spin mode is possible.

The dynamically polarized, frozen spin target at MAMI was constructed for use inside the Crystal Ball detector with beams of tagged photons. When being polarized the cryostat is moved outside of the Crystal Ball. Thin superconducting holding coils were installed on the thermal radiation protection shields of the refrigerator to maintain the target polarization during the experiments. Details of the frozen spin target at MAMI can be found, e.g. in Ref. [1].

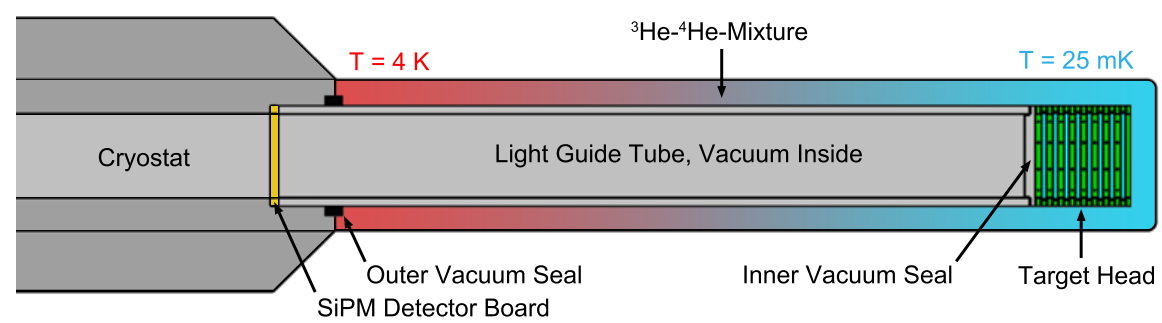

Figure 1: Schematics of the active polarized proton target. The target is immersed in a liquid ${ }^{3} \mathrm{He} /{ }^{4} \mathrm{He}$ mixture with a temperature of $T \sim 25 \mathrm{mK}$ at the target head. This design includes wavelength-shifting material to transport light from the scintillators in the target head to the glass tube which is read out at the warm side by SiPMs.

An active polarized proton target is being developed to identify the reactions below the pion threshold by detecting recoil protons inside the Mainz-Dubna dilution cryostat [2]. Polarizable plastic scintillator disks are stacked in a target head made of wavelength-shifting material or 
PMMA. The scintillation light is guided through a tube of borosilicate glass to silicon photomultipliers (SiPMs). All parts of the active polarized proton target are operated at cryogenic temperatures.

If the SiPMs are directly connected to the scintillator disks in the active target those would have to withstand temperatures in the $\mathrm{mK}$ regime and would insert an intolerable large amount of heat into the cryostat. Because of a large temperature gradient in the $2 \mathrm{~m}$ long cryostat the SiPMs can be placed downstream in a temperature region near $T \sim 100 \mathrm{~K}$. Then the scintillation light has to be guided from the target head to the read-out region and the electric signals have to be transmitted from that region to the outside of the cryostat. In a beam-time at MAMI the separation of recoil protons from electromagnetic background reactions was demonstrated with such a design reaching a signal-to-background ratio of five.

\section{Design and fabrication of the target head}

The target head includes several disks of solid polarizable scintillator. Recoiling protons can be detected inside the target material. The stack geometry ensures an effective cooling of the material inside the liquid helium of the mixing chamber. Test under cryogenic conditions have been done to check the relaxation times and maximum polarization of radical doped scintillating polystyrene material provided by the University of Massachusetts, Amherst. A maximum degree of polarization of the order of $70 \%$ could be reached. The light transport to the SiPMs is done by a hollow borosilicate glass tube of $26 \mathrm{~mm}$ outer diameter and $20.4 \mathrm{~mm}$ inner diameter. A scheme is shown in Fig. 1. The tube is immersed in liquid helium, the inside of the tube being in vacuum. An inner vacuum seal closes the tube at the target head, an outer vacuum seal connects the tube to the cryostat where the SiPMs are mounted on a circuit board.

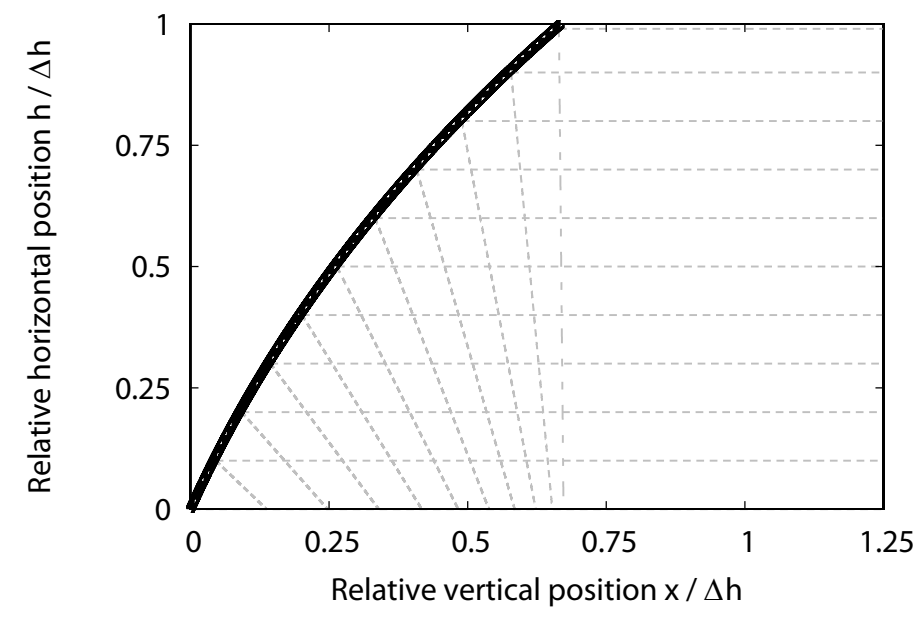

Figure 2: Parametrized model of the axially symmetric target head shape that was used in the computer aided fabrication using a CNC mill. The connection to the borosilicate glass tube used as a light guide is on the $x$-axis, the light from the scintillator arrives on the $h$-axis from the right side. The shape was optimized for maximum light transmission to the light guide making use of the angle of total reflection in borosilicate. A target head using this "mirror" design was realized with a length of $\Delta h=20 \mathrm{~mm}$. 

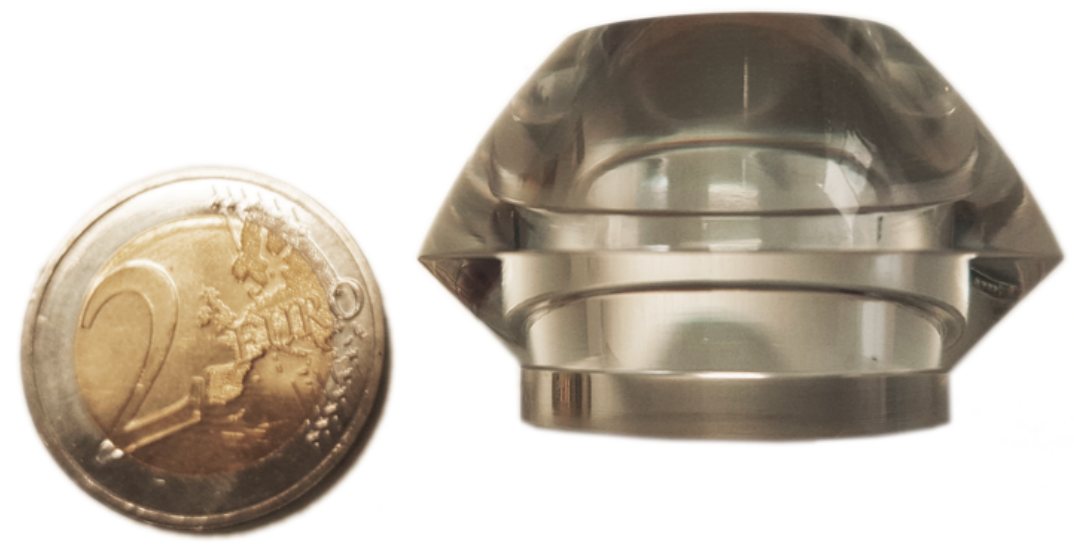

Figure 3: Photograph of the light concentrator which is part of the target head in the "mirror" design. The length of the concentrator is $\Delta h=20 \mathrm{~mm}$. The neighboring coin sets the scale.
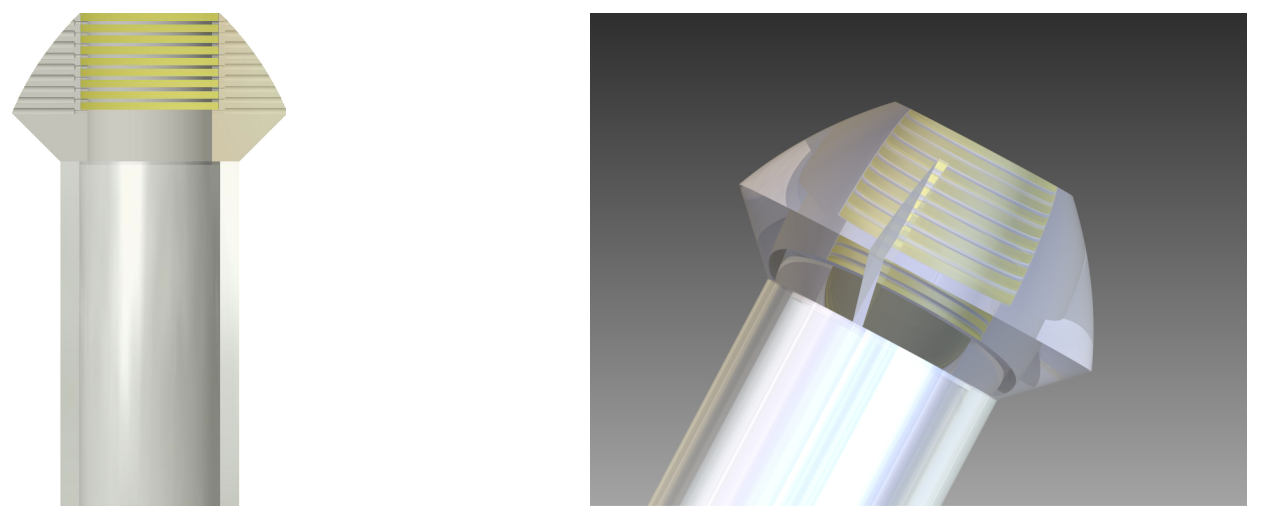

Figure 4: Left: cross-sectional view of the axially symmetric target head with nine scintillator disks (yellow) and the attached borosilicate glass tube guiding the scintillator light in vertical direction. Right: rendering of the target head. The outer and inner diameters of the glass tube are $26 \mathrm{~mm}$ and $20.4 \mathrm{~mm}$, respectively. The diameter and thickness of the solid scintillator disks are $20 \mathrm{~mm}$ and $1 \mathrm{~mm}$, respectively.

The favored design of the target includes an axially symmetric specifically shaped light concentrator made from PMMA that guides the scintillation light by total reflection at the PMMA-He surface from the disks to the glass tube. The shape was optimized for maximum light transmission to the light guide leading to a surface contour in the cross-section of the concentrator that follows the curve

$$
h(x) / \Delta h=4 /(\pi-2 \alpha)\left(\cos ^{-1}[\cos (\alpha / 2) \cdot \exp (-(\pi / 4-\alpha / 2) \cdot x / \Delta h)]-\alpha / 2\right)
$$

where $\alpha$ is the angle of total reflection. A model of the light transport inside the concentrator is shown in Fig. 2. The parametrization of the geometry was used in the computer aided fabrication of the light concentrator using a CNC mill in the mechanical workshop, which put challenging 
demands on the processing. A target head using this "mirror" design was realized with a length of $\Delta h=20 \mathrm{~mm}$. A photograph of the light concentrator is shown in Fig. 3. The complete target head, including the scintillator disks, the light concentrator, and part of the light guide is shown in Fig. 4.

An alternative design of the target head uses the wavelength-shifting material BC499-53 to transport light from the scintillators to the borosilicate glass tube. In this design the wavelengthshifting material is surrounding the scintillating disks and transforms the blue light directed to the outside of the target head into green light that is emitted isotropically.

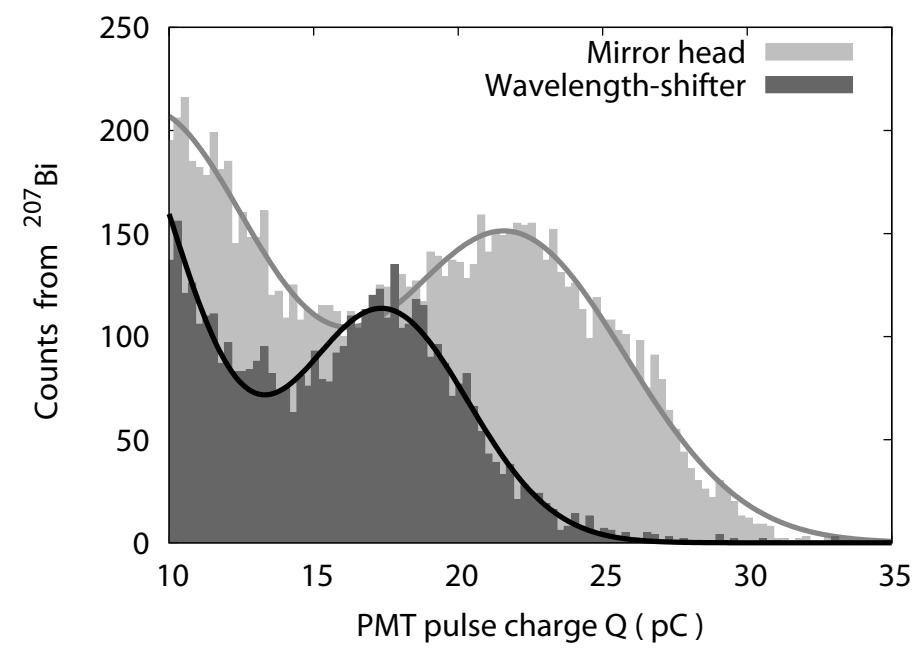

Figure 5: Comparison of two different target head designs. The light yield from a mono-energetic ${ }^{207} \mathrm{Bi}$ source in front of the target head under study was determined with a photomultiplier and ADC read-out. The results show an increase by $25 \%$ in light yield of the mirror head design over the wavelength-shifter design.

Both designs were realized and studied in a laboratory tests. The light yield from a monoenergetic ${ }^{207} \mathrm{Bi}$ source in front of the target head under study was determined with a photomultiplier and ADC read-out. The results show an increase by $25 \%$ in light yield of the mirror head design over the wavelength-shifter design, see Fig. 5. The increased light yield and the omission of the second fluorescence process in the wavelength-shifter will also improve the time resolution of the detector.

\section{Design and characterization of the light guiding tube}

The borosilicate glass tube is used as light guide from the target head to the photo-sensors and serves the purpose of placing the SiPMs at a warmer position inside the cryostat. The required features of the tube are (i) super-fluid helium tightness, (ii) high stability against thermal stress, (iii) low thermal conductivity at cryogenic temperatures, and (iv) good visible light transmission.

Fig. 6 shows the measured light intensity attenuation for several borosilicate glass tubes using a setup with a LED pulser and a PMT read-out. A single exponential fit to the data points returns an attenuation length of $\Lambda=607 \pm 3 \mathrm{~mm}$ in the range of $0.5-1.5 \mathrm{~m}$.

The thermal conductivity of the glass is low and has a small variation with temperature, while the alternatives, sapphire or quartz, show a variation over several orders of magnitude when cooling 


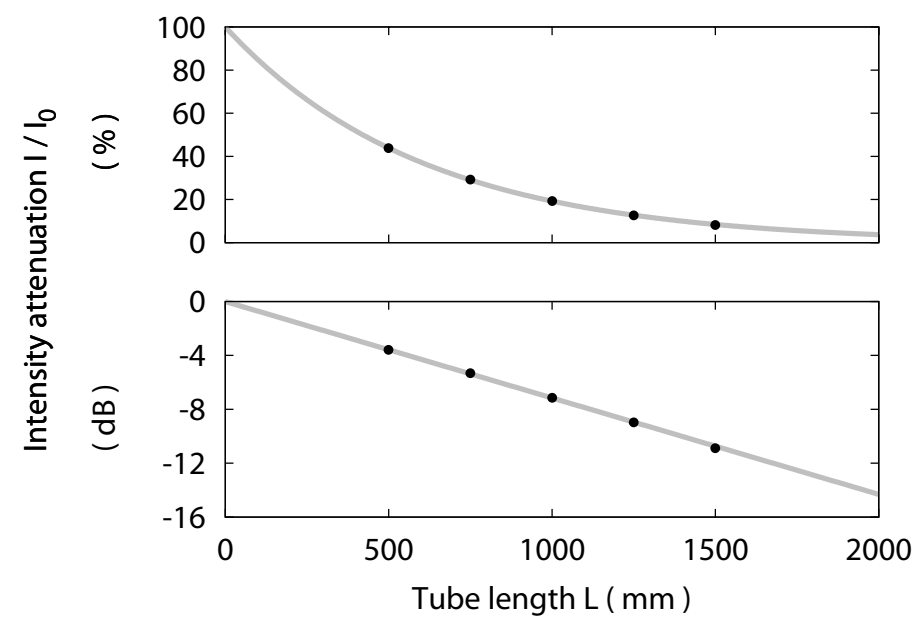

Figure 6: Measured light intensity attenuation in the borosilicate glass tube used as light guide from the target head to the photo-sensors. A single exponential fit with an attenuation length of $\Lambda=607 \pm 3 \mathrm{~mm}$ can be used to describe the attenuation in the range of $0.5-1.5 \mathrm{~m}$.

from room temperature to below $10 \mathrm{~K}$. The thermal expansion coefficient of borosilicate, $\lambda \approx$ $3.3 \times 10^{-6} \mathrm{~K}^{-1}$, puts little stress on the tube. Repeated tests have shown that the tube can withstand the immediate cooling from $300 \mathrm{~K}$ down to $77 \mathrm{~K}$ and the evacuation to $7 \times 10^{-10} \mathrm{mbar}$.

\section{Design of the optical readout system}

The photo-sensors will cover the outside of the tube in a ring on a printed circuit board. The required features of the detectors are (i) high gain, (ii) high rate capability, (iii) small surface mount type, and last but not least (iv) good operational performance at cryogenic temperatures. SiPM operation at cryogenic temperatures fails for many common devices, some show dramatic changes in the pulse shape by acquiring slow components, see e.g. [3]. A particular type MAPD-3N from Zecotek [4] with deep channels in the silicon substrate instead of quenching resistors was tested at liquid helium temperature and many other SiPMs have been studied extensively at cryogenic temperatures $[5,6]$.

15 SiPMs of size $3 \times 3 \mathrm{~cm}^{2}$ by SensL [7] will be used on a ring-shaped circuit board. The SensL sensors feature low dark count rates and have shown a very good performance at liquid nitrogen and liquid helium temperatures. The active area of the sensors is covering $60 \%$ of the tube cross-section in the current design.

\section{Summary}

High-precision double polarized photo-production experiments off the proton can profit very much from an active polarized proton target. Examples are real Compton scattering off polarized protons or the study of the Gerasimov-Drell-Hearn sum rule. A target head made from polarizable scintillating disks positioned inside the Mainz-Dubna cryostat and read out by SiPMs connected by a borosilicate tube to the target head has been developed. 


\section{Acknowledgments}

The authors express their gratitude to all scientists, engineers, and technicians at Mainz University who helped with the technical challenges experienced during the development of the target. This work was supported in part by Deutsche Forschungsgemeinschaft (SFB 1044) and National Science Foundation Grant No. PHY-1309130 \& IIA-1358175.

\section{References}

[1] A. Thomas, Eur. Phys. J. ST 198, 171 (2011)

[2] M. Biroth, Diploma thesis, Johannes Gutenberg-Universität, Mainz (2014)

[3] H. Otono, Study of MPPC at liquid nitrogen temperature, in International Workshop on new Photon-Detectors, 27 - 29 June 2007, Kobe, Japan (2007), Proceedings of Science (PD07), p. 007, http://pos.sissa.it/archive/conferences/051/007/PD07_007.pdf

[4] Zecotek MAPD White Paper, Zecotek Photonics, Richmond, Canada (2011), http://www. zecotek.com/media/MAPD-WhitePaper-March-2011.pdf

[5] M. Biroth, P. Achenbach, E. Downie, A. Thomas, Nucl. Instrum. Methods Phys. Res. A 787, 68 (2015)

[6] P. Achenbach, M. Biroth, E. Downie, A. Thomas, Nucl. Instrum. Methods Phys. Res. A (2015), http://dx.doi.org/10.1016/j.nima.2015.10.091

[7] C-Series Low Noise, Blue-Sensitive Silicon Photomultipliers, SensL Technologies, Cork, Ireland (2015), http://sensl.com/downloads/ds/DS-MicroCseries.pdf 